\title{
Covariance-Based OFDM Spectrum Sensing with Sub-Nyquist Samples
}

\author{
Alireza Razavi ${ }^{\dagger}$, Mikko Valkama $^{\dagger}$, Danijela Cabric ${ }^{\ddagger}$ \\ ${ }^{\dagger}$ Tampere University of Technology, Tampere, Finland \\ $\ddagger$ University of California, Los Angeles (UCLA), USA \\ email: alireza.razavi@tut.fi,mikko.e.valkama@tut.fi,danijela@ee.ucla.edu
}

\begin{abstract}
In this paper, we propose a feature-based method for spectrum sensing of OFDM signals from sub-Nyquist samples over a single band. We exploit the structure of the covariance matrix of OFDM signals to convert an underdetermined set of covariancebased equations to an overdetermined one. The statistical properties of sample covariance matrix are analyzed and then based on that an approximate Generalized Likelihood Ratio Test (GLRT) for detection of OFDM signals from sub-Nyquist samples is derived. The method is also extended to the frequency-selective channels.
\end{abstract}

\section{Introduction}

Cognitive Radio (CR) is emerging as a promising technology for improving the efficiency of radio spectrum use in wireless communication systems (Mitola and Maguire, 1999). Spectrum sensing (SS) is the most vital task in CR defined as identifying spectrum holes by 
sensing the radio spectrum and utilizing them without causing interference to primary users (PUs) (Havkin et al., 2009). Of special interest in this regard is sensing of OFDM signals (Chaudhari et al., 2009; Axell and Larsson, 2011). OFDM is one of the most effective multicarrier techniques for broadband wireless communications which is employed by many of the current and emerging wireless technologies.

On the other hand, due to the limitations of today's analog-to-digital converter (ADC) circuits which cannot support very high bandwidth and need excessive memory and prohibitive energy costs for implementing digital signal processing systems (Cohen et al.,, 2011), it may be very costly and even impractical to sense the signal based on Nyquist-rate samples. This has motivated researchers to study sub-Nyquist methods for wideband spectrum sensing in CR networks; see, e.g., (Mishali and Eldar, 2011; Tian and Giannakis, 2007; Tian et al., 2012; Polo et al., 2009; Tian, 2011; Leus and Tian, 2011; Ariananda and Leus, 2011; Cohen et al., 2011; Sun et al., 2012; Rebeiz et al., 2012). But to the best of our knowledge, there has been less efforts targeting the detection of OFDM signals from sub-Nyquist samples. In this paper, we propose a new feature-based approach for sensing an OFDM signal occupying a single band from sub-Nyquist samples.

Related research and previous work: The problem of OFDM sensing using secondorder statistics has been already studied in, e.g., (Chaudhari et al., 2009; Axell and Larsson, 2011; Bokharaiee et al., 2011; Al-Habashna et al., 2012). All of these methods in some way exploit the correlation induced by CP to sense the presence of OFDM signal, but perform the detection based on Nyquist rate samples which, as discussed earlier, might need expensive ADCs espeially for wideband signals.

During the past several years, the problem of spectrum sensing from sub-Nyquist samples has attracted a lot of attention. While many of the approaches proposed so far rely on the sparsity in the frequency domain arising from spectrum underutilization Tian and Giannakis (2007); Tian (2008); Polo et al. (2009); Sun et al. (2013), there has also been methods which 
do not necessarily need sparseness in the spectrum to work Tian et al. (2012); Tian (2011); Leus and Tian (2011); Ariananda and Leus (2011); Razavi et al. (2013); Cohen et al. (2011); Rebeiz et al. (2012). For instance, the methods proposed in Tian et al. (2012); Tian (2011); Leus and Tian (2011); Cohen et al. (2011); Rebeiz et al. (2012); Razavi et al. (2013) are based on recovering the Spectral Correlation Function (SCF) of the signal from sub-Nyquist samples. All of these methods need sparsity in the SCF for detecting the signal. Since the SCF matrix of the Nyquist-sampled OFDM signal (see, e.g., (Tian et al., 2012, Equation $(10))$ ) is nonzero everywhere, then it is not possible to use these methods for recovering the SCF of OFDM signals from sub-Nyquist samples as they need sparsity in the SCF for recovering it using Compressive Sensing (CS) algorithms or converting the underdetermined set of equations to an overdetermined one. The methods proposed in Ariananda and Leus (2011) and (Tian et al., 2012, Section IV) reconstruct the Power Spectral Density (PSD) from sub-Nyquist samples and then decide on the presence of signal based on the recovered PSD. Similar to conventional energy detection methods Yucek and Arslan (2009), the main challenges with these methods are selecting the threshold and poor performance in low signal-to-noise ratios. In Razavi et al. (2013), we detected an OFDM signal over a single band from sub-Nyquist samples. Although this method assume that the whole band is occupied by the OFDM signal, but we inject the sparsity to SCF using a cyclostationary signature Adrat et al. (2009); Sutton et al. (2008) embedded in the signal to assist with the detection procedure. This might be of help in the problem of rendezvous and cognitive network identification Razavi et al. (2014), but it cannot be used in detecting primary users, as primary users usually do not care about secondary users and therefore do not assist them via injecting a signature in their own signals to facilitate the spectrum sensing task.

Our contribution: In this paper, we propose a new feature-based approach for sensing OFDM signals from sub-Nyquist samples over a single band entirely occupied by the signal. In other words, we do not assume any sparsity in the spectrum. Instead, the method exploits the features of covariance matrix of OFDM signals stemming from the insertion of 
cyclic prefix (CP). These features, as we will show later, will help us to convert the underdetermined set of two-dimensional (2-D) equations which relates the available covariance matrix of sub-Nyquist samples to the unavailable covariance matrix of Nyquist samples, to an overdetermined one-dimensional (1-D) one. Then, based on the statistical properties of the sample covariance, an approximate GLRT-based detector is derived. The method is also extended to the case of frequency-selective channels.

Paper organization: The rest of the paper is organized as follows. In Section 2 the system model over Gaussian channels is given and the problem is formulated. In Section 3 the relevant statistical properties of sample covariance matrix are studied and a covariancedomain linear system is derived. The approximate GLRT-based detector is introduced and formulated in Section 4. In Section 5 we extend the results to the frequency-selective channel case. Finally, we study the performance of the proposed method by simulation experiments in Section 6. Conclusions are drawn in Section 7 and some details of the derivations are given in the Appendix.

Notations and Mathematical Preliminaries: Throughout this paper matrices and vectors are denoted by capital and small boldface letters, respectively. = denotes the equality and $\triangleq$ denotes the definition. $\mathbb{E}$ is reserved for statistical expectation operator, $\operatorname{Cov}(\mathbf{x}, \mathbf{y})$ represents the covariance matrix between random vectors $\mathbf{x}$ and $\mathbf{y}$, and $\otimes$ denotes the Kronecker product. $\mathbf{I}_{P}$ and $\mathbf{0}_{P, Q}$ represent, respectively, $P \times P$ identity matrix and $P \times Q$ full-zero matrix. For an arbitrary $M \times N$ matrix $\mathbf{A},[\mathbf{A}]_{i, j}$ denotes the $(i, j)$-th entry of the matrix and $\mathbf{a}_{i}, i=1,2, \ldots, N$ denotes its $i$-th column. $\operatorname{vec}(\mathbf{A})$ is the vectorization of $\mathbf{A}$ defined as $\operatorname{vec}(\mathbf{A}) \triangleq\left[\mathbf{a}_{1}^{T}, \ldots, \mathbf{a}_{N}^{T}\right]^{T}$. Furthermore, if $M=N$, then $\operatorname{vech}(\mathbf{A})$, known as half-vectorization of $\mathbf{A}$, is the $\frac{N(N+1)}{2} \times 1$ vector which is obtained by column-wise stacking of only the elements on and below the main diagonal. It can be proven that for an $N \times N$ matrix $\mathbf{A}$ (Seber, 2008, Chapter 11)

$$
\operatorname{vech}(\mathbf{A})=\mathbf{\Omega}_{N} \operatorname{vec}(\mathbf{A})
$$


where

$$
\boldsymbol{\Omega}_{N} \triangleq\left[\begin{array}{ccccc}
\mathbf{I}_{N}^{(0)} & \mathbf{0}_{N, N} & \mathbf{0}_{N, N} & \ldots & \mathbf{0}_{N, N} \\
\mathbf{0}_{N-1, N} & \mathbf{I}_{N}^{(1)} & \mathbf{0}_{N-1, N} & \ldots & \mathbf{0}_{N-1, N} \\
\mathbf{0}_{N-2, N} & \mathbf{0}_{N-1, N} & \mathbf{I}_{N}^{(2)} & \ldots & \mathbf{0}_{N-2, N} \\
\vdots & \vdots & \vdots & \ddots & \vdots \\
\mathbf{0}_{1, N} & \mathbf{0}_{1, N} & \mathbf{0}_{1, N} & \ldots & \mathbf{I}_{N}^{(N-1)}
\end{array}\right]
$$

where $\mathbf{I}_{N}^{(i)}$ denotes an identity matrix whose first $i$ rows are discarded. Remark that $\mathbf{I}_{N}^{(0)}=\mathbf{I}_{N}$. Moreover, if $\mathbf{A}$ is a symmetric matrix, then (Seber, 2008, Chapter 11)

$$
\operatorname{vec}(\mathbf{A})=\boldsymbol{\Gamma}_{N} \operatorname{vech}(\mathbf{A})
$$

where $\boldsymbol{\Gamma}$ is an $\left(N^{2}\right) \times(N(N+1) / 2)$ matrix with entries $((i-1) N+j,(j-1)(N-j / 2)+i)$ and $((j-1) N+i,(j-1)(N-j / 2)+i)$ for $1 \leq j \leq i \leq N$ equal to 1 and the rest of elements equal to zero. Remark that $\boldsymbol{\Omega}_{N} \boldsymbol{\Gamma}_{N}=\mathbf{I}_{N(N+1) / 2}$.

Furthermore, for any three arbitrary matrices $\mathbf{A}, \mathbf{B}$, and $\mathbf{C}$ of suitable sizes, we have the following two equalities (Seber, 2008, Chapter 11)

$$
\operatorname{vec}\left(\mathbf{A B C} \mathbf{C}^{T}\right)=(\mathbf{C} \otimes \mathbf{A}) \operatorname{vec}(\mathbf{B})
$$

and

$$
\mathbf{A B C}^{T}=\sum_{i} \sum_{j}[\mathbf{B}]_{i, j} \mathbf{a}_{i} \mathbf{c}_{j}^{T}
$$

where $\mathbf{a}_{i}$ and $\mathbf{c}_{j}$ are $i$-th and $j$-th columns of $\mathbf{A}$ and $\mathbf{C}$, respectively. 


\section{System Model over Gaussian Channels}

Consider a secondary user (SU) with the goal to detect the presence of an OFDM signal. Denoting the received signal by $x(t)$, we can formulate the problem as deciding between the following two hypotheses

$$
\left\{\begin{array}{l}
\mathcal{H}_{0}: x(t)=n(t) \\
\mathcal{H}_{1}: x(t)=s(t)+n(t)
\end{array}\right.
$$

where $s(t)$ is an OFDM signal and $n(t) \sim \mathcal{C N}\left(0, \sigma_{n}^{2}\right)$. If the number of subcarriers is large enough, from central limit theory we have $s(t) \sim \mathcal{C N}\left(0, \sigma_{s}^{2}\right)$. Suppose next that we have sampled the signal $x(t)$ at a sub-Nyquist rate to collect compressive samples $z(t)$. Matrixwise, this can be described as 1

$$
\mathbf{z}(k)=\mathbf{A x}(k), k=1,2, \ldots, N_{B}
$$

where $\mathbf{z}(k) \triangleq[z[k M], z[k M+1], \ldots, z[k M+M-1]]^{T}$ consists of sub-Nyquist (compressive) samples, $\mathbf{x}(k) \triangleq[x[k N], x[k N+1], \ldots, x[k N+N-1]]^{T}$ consists of (unavailable) Nyquist samples, $N_{B}$ is the total number of taken frames, and $\mathbf{A}$ is the $M \times N$ real-valued measurement matrix. $\rho \triangleq \frac{M}{N}<1$ is called compression (or downsampling) ratio.

Now, the task of a sub-Nyquist OFDM detector is to decide whether the OFDM signal exists or not, based on sub-Nyquist measurements $\{\mathbf{z}(k)\}_{k=1}^{N_{B}}$.

It can be easily seen that $\mathbf{R}_{z}=\mathbf{A} \mathbf{R}_{x} \mathbf{A}^{T}$ where the $N \times N$ matrix $\mathbf{R}_{x} \triangleq \mathbb{E}\left(\mathbf{x}(k) \mathbf{x}^{H}(k)\right)$ and $M \times M$ matrix $\mathbf{R}_{z} \triangleq \mathbb{E}\left(\mathbf{z}(k) \mathbf{z}^{H}(k)\right)$ are covariance matrices of $\mathbf{x}$ and $\mathbf{z}$, respectively. Let us denote the useful symbol length of the considered OFDM signal by $T$, the cyclic prefix

\footnotetext{
${ }^{1}$ We remark here that there are two main strategies for sub-Nyquist sampling of a signal Mishali et al. (2011): Random Demodulator (RD), also known as Analog-to-Information Converter (AIC), Tropp et al. (2010); Kirolos et al. (2006), and Modulated Wideband Converter (MWC) Mishali and Eldar (2010). Both of these strategies can be mathematically formulated as in (7) Mishali et al. (2011).
} 
length by $T_{c p}$ and the total OFDM symbol length by $T_{s y m}=T+T_{c p}$ which are all assumed to be known to the cognitive user. Assume that the basic sample duration is normalized to 1. Then by setting $N=T_{\text {sym }}$, matrix $\mathbf{R}_{x}$ of the noisy OFDM signal (i.e. $x(t)$ under $\mathcal{H}_{1}$ ) can be written as

$$
\left[\mathbf{R}_{x}\right]_{i, j}= \begin{cases}\sigma_{s}^{2}+\sigma_{n}^{2} & \text { if } i=j \\ \sigma_{s}^{2} & \text { if }|i-j|=T \\ 0 & \text { otherwise }\end{cases}
$$

On the other hand, under $\mathcal{H}_{0}$ we have

$$
\left[\mathbf{R}_{x}\right]_{i, j}=\left\{\begin{array}{cl}
\sigma_{n}^{2} & \text { if } i=j, \\
0 & \text { otherwise. }
\end{array}\right.
$$

To encompass the descriptions of $\mathbf{R}_{x}$ under $\mathcal{H}_{0}$ and $\mathcal{H}_{1}$, we present it by

$$
\mathbf{R}_{x}=\tau_{0} \mathbf{I}_{N}+\tau_{s} \boldsymbol{\Lambda},
$$

where $\mathbf{I}_{N}$ is the $N \times N$ identity matrix and $\boldsymbol{\Lambda}$ is defined as

$$
[\boldsymbol{\Lambda}]_{i, j} \triangleq \begin{cases}1 & \text { if }|i-j|=T \\ 0 & \text { otherwise }\end{cases}
$$

and then distinguish between $\mathcal{H}_{0}$ and $\mathcal{H}_{1}$ as

$$
\left\{\begin{array}{l}
\mathcal{H}_{0}:\left(\tau_{0}, \tau_{s}\right)=\left(\sigma_{n}^{2}, 0\right), \\
\mathcal{H}_{1}:\left(\tau_{0}, \tau_{s}\right)=\left(\sigma_{n}^{2}+\sigma_{s}^{2}, \sigma_{s}^{2}\right) .
\end{array}\right.
$$


Since in both cases of (12) we have $\tau_{0}=\tau_{s}+\sigma_{n}^{2}$, therefore (12) can be simplified as

$$
\left\{\begin{array}{l}
\mathcal{H}_{0}: \tau_{s}=0, \\
\mathcal{H}_{1}: \tau_{s} \neq 0
\end{array}\right.
$$

In other words, the problem of detection of OFDM signal can be re-expressed as identifying whether in the general description of $\mathbf{R}_{x}$ in (10) the parameter $\tau_{s}$ is zero or not.

In practice, covariance matrices are not readily available and hence we substitute them by

sample covariance matrices $\overline{\mathbf{R}}_{x} \triangleq \frac{1}{N_{B}} \sum_{k=1}^{N_{B}} \mathbf{x}(k) \mathbf{x}^{H}(k)$ and $\overline{\mathbf{R}}_{z}=\frac{1}{N_{B}} \sum_{k=1}^{N_{B}} \mathbf{z}(k) \mathbf{z}^{H}(k)$, where $N_{B}$ is the number of frames used for the computations. It is easy to see that

$$
\overline{\mathbf{R}}_{z}=\mathbf{A} \overline{\mathbf{R}}_{x} \mathbf{A}^{T} .
$$

In the next sections we will show how the sub-Nyquist sample covariance matrix $\overline{\mathbf{R}}_{z}$ can be used to perform the hypothesis testing in (6) based on the observation made in (13).

\section{Covariance-Based Linear Equations for OFDM De- tection}

The sample covariance matrix $\overline{\mathbf{R}}_{x}$ can in general be written as the sum of covariance matrix $\mathbf{R}_{x}$ and an error term $\mathbf{W}$ stemming from the finite-sample effects. From now on, we call $\mathbf{W}$ the finite-sample noise and express this as

$$
\overline{\mathbf{R}}_{x}=\mathbf{R}_{x}+\mathbf{W} .
$$

It is easy to verify that $\mathbb{E}_{x}\left(\overline{\mathbf{R}}_{x}\right)=\mathbf{R}_{x}$ under both $\mathcal{H}_{0}$ and $\mathcal{H}_{1}$, and therefore conclude that $\mathbb{E}_{x}(\mathbf{W})=$ 0. Furthermore, from (Goldberger, 1991, Chapters 9-10) we know that the entries 
of $\mathbf{W}$ have asymptotic Normal distribution. The following theorem then states the covariance of entries of W. Parts of this theorem can also be found in Axell and Larsson (2011).

Theorem 1. Suppose that $w_{i, j}$ and $w_{p, q}$ are two arbitrary entries of $\mathbf{W}$ below or on main diagonal (i.e. $i \geq j, p \geq q$ ). Then

$$
\mathbb{E}\left(w_{i, j} w_{p, q} \mid \mathcal{H}_{0}\right)= \begin{cases}\frac{\tau_{0}^{2}}{2 N_{B}} & \text { if }(i, j)=(p, q) \\ 0 & \text { otherwise, }\end{cases}
$$

where $\tau_{0} \triangleq \sigma_{n}^{2}$, and

$$
\mathbb{E}\left(w_{i, j} w_{p, q} \mid \mathcal{H}_{1}\right)= \begin{cases}\frac{\tau_{0}^{2}}{N_{B}} & \text { if }(i, j)=(p, q), i-j=0, \\ \frac{\tau_{s}^{2}+\tau_{0}^{2}}{2 N_{B}} & \text { if }(i, j)=(p, q), i-j=T, \\ \frac{\tau_{0}^{2}}{2 N_{B}} & \text { if }(i, j)=(p, q), i-j \notin\{0, T\} \\ \frac{\tau_{0} \tau_{s}}{N_{B}} & \text { if } i=j=p=q+T \\ 0 & \text { otherwise, }\end{cases}
$$

where $\tau_{0} \triangleq \sigma_{n}^{2}+\sigma_{s}^{2}$ and $\tau_{s} \triangleq \sigma_{s}^{2}$.

Proof. Proof is deferred to Appendix.

Inserting next (15) in (14) yields

$$
\overline{\mathbf{R}}_{z}=\mathbf{A R}_{x} \mathbf{A}^{T}+\mathbf{A W A}^{T}
$$

Notice that since $\mathbf{R}_{x}$ and $\mathbf{A}$ are both real-valued, the signal part of (18) is real-valued and the imaginary part only includes the effect of finite-sample error W. In fact, we can simply change (18) to a real-valued equation by keeping only the real part of $\overline{\mathbf{R}}_{z}$ and throwing away the uninformative imaginary part. To avoid introducing extra notations, from now on we 
assume that (18) represents a real-valued matrix equation.

The problem of sensing OFDM signal can then be restated as testing whether in 2-D linear model (18) we have $\mathbf{R}_{x}=\tau_{0} \mathbf{I}$ or $\mathbf{R}_{x}=\tau_{0} \mathbf{I}+\tau_{s} \boldsymbol{\Lambda}$ for some nonzero unknown parameters $\tau_{0}$ and $\tau_{s}$. The first problem in this regard is that (18) represents an underdetermined linear system of equations. To solve this issue, we first apply the vech operator to (18) and use (1), (3), (41) and (10) to obtain

$$
\overline{\mathbf{r}}_{z}=\boldsymbol{\Omega}_{M}(\mathbf{A} \otimes \mathbf{A})\left(\tau_{0} \operatorname{vec}(\mathbf{I})+\tau_{s} \operatorname{vec}(\boldsymbol{\Lambda})\right)+\boldsymbol{\Omega}_{M}(\mathbf{A} \otimes \mathbf{A}) \boldsymbol{\Gamma}_{N} \mathbf{w}
$$

where $\overline{\mathbf{r}}_{z}=\operatorname{vech}\left(\overline{\mathbf{R}}_{z}\right)$ and $\mathbf{w}=\operatorname{vech}(\mathbf{W})$. From (44) and (5) it is easy to verify that $(\mathbf{A} \otimes$ $\mathbf{A}) \operatorname{vec}(\mathbf{I})=\sum_{i=1}^{N}\left(\mathbf{a}_{i} \otimes \mathbf{a}_{i}\right)$ and $(\mathbf{A} \otimes \mathbf{A}) \operatorname{vec}(\boldsymbol{\Lambda})=\sum_{i=T+1}^{N}\left(\mathbf{a}_{i-T} \otimes \mathbf{a}_{i}+\mathbf{a}_{i} \otimes \mathbf{a}_{i-T}\right)$ and therefore rewrite (19) as

$$
\begin{aligned}
\overline{\mathbf{r}}_{z} & =\tau_{s} \mathbf{b}_{s}+\tau_{0} \mathbf{b}_{0}+\mathbf{v} \\
& =\mathbf{B} \boldsymbol{\theta}+\mathbf{v}
\end{aligned}
$$

where $\mathbf{b}_{0} \triangleq \boldsymbol{\Omega}_{M} \sum_{i=1}^{N}\left(\mathbf{a}_{i} \otimes \mathbf{a}_{i}\right)$ and $\mathbf{b}_{s} \triangleq \boldsymbol{\Omega}_{M} \sum_{i=T+1}^{N}\left(\mathbf{a}_{i-T} \otimes \mathbf{a}_{i}+\mathbf{a}_{i} \otimes \mathbf{a}_{i-T}\right), \mathbf{B} \triangleq\left[\mathbf{b}_{0} \mathbf{b}_{s}\right]$, $\boldsymbol{\theta} \triangleq\left[\tau_{0} \tau_{s}\right]^{T}, \mathbf{v} \triangleq \mathbf{K w}$ is the additive noise vector, and $\mathbf{K} \triangleq \boldsymbol{\Omega}_{M}(\mathbf{A} \otimes \mathbf{A}) \boldsymbol{\Gamma}_{N}$.

Now, the problem of OFDM signal detection can be expressed as testing whether in the classical linear model (20) the second entry of parameter vector $\boldsymbol{\theta}$ is zero or not. We formulate this in the next section.

We remark here that since in linear regression equation (20) the number of unknown parameters is two and the number of equations is $M(M+1) / 2$, any $M \geq 2$ is theoretically enough for estimating $\tau_{s}$ and performing the test. 


\section{Approximate Generalized Likelihood Ratio Test}

To derive the GLRT-based detector, we first notice that the noise vector $\mathbf{v}$ in (20) is not white. Therefore the first step is to whiten the noise by multiplying both sides of (20) by $\Sigma_{v}^{-1 / 2}$, where

$$
\Sigma_{v}=\mathbf{K} \boldsymbol{\Sigma}_{w} \mathbf{K}^{T}
$$

and $\boldsymbol{\Sigma}_{w}$ is the covariance matrix of $\mathbf{w}=\operatorname{vech}(\mathbf{W})$. Remark that as proved in Theorem $1, \boldsymbol{\Sigma}_{w}$, and therefore $\boldsymbol{\Sigma}_{v}$, does not expose the same expression under $\mathcal{H}_{0}$ and $\mathcal{H}_{1}$. Hence, to simplify the test, in this section we formulate an GLRT-based detector based on approximating $\Sigma_{v}$ with a hypothesis-independent covariance matrix which compromises the properties of covariance matrices under the two hypotheses.

We first adopt a hypothesis-independent approximation for the entries of $\boldsymbol{\Sigma}_{w}$ as follows

$$
\mathbb{E}\left(w_{i, j} w_{p, q}\right)= \begin{cases}2 c & \text { if }(i, j)=(p, q), i-j=0 \\ c & \text { if }(i, j)=(p, q), i-j \neq 0 \\ 0 & \text { otherwise }\end{cases}
$$

where $c$ is an unknown constant. Remark that $\mathbb{E}\left(w_{i, j} w_{p, q}\right)$ in (22) shares properties with both $\mathbb{E}\left(w_{i, j} w_{p, q} \mid \mathcal{H}_{0}\right)$ in (16) and $\mathbb{E}\left(w_{i, j} w_{p, q} \mid \mathcal{H}_{1}\right)$ in (17): like (16) it is zero for $(i, j) \neq(p, q)$, but then for $(i, j)=(p, q)$ it is like (17) with $\tau_{s} \ll \tau_{0}$. Equation (22) then implies that $\boldsymbol{\Sigma}_{w}$ has the following form

$$
\Sigma_{w}=c \Delta
$$

where $\boldsymbol{\Delta}$ is an $\frac{N(N+1)}{2}$ diagonal matrix with diagonal elements in locations $1,1+(N)$, $1+(N)+(N-1), 1+(N)+(N-1)+(N-2), \ldots$ being equal to 2 and the rest are equal to 1 . In fact, the diagonal elements having value 2 correspond to the first case of (22) and 
the rest correspond to its second case. The third case of (22) implies that $\Sigma_{w}$ is diagonal.

Now, multiplying both sides of (20) by $\boldsymbol{\Gamma}=\left(\mathbf{K} \boldsymbol{\Delta} \mathbf{K}^{T}\right)^{-1 / 2}$ yields:

$$
\tilde{\mathbf{r}}_{z}=\tilde{\mathbf{B}} \boldsymbol{\theta}+\tilde{\mathbf{v}}
$$

where $\tilde{\mathbf{r}}_{z} \triangleq \Gamma \overline{\mathbf{r}}_{z}, \tilde{\mathbf{B}} \triangleq \Gamma \mathbf{B}$, and $\tilde{\mathbf{v}} \triangleq \Gamma \mathbf{v}$. Based on this, the problem of OFDM detection can be expressed as doing hypothesis testing problem (13) for linear system (24) in which the additive noise has distribution $\tilde{\mathbf{v}} \sim \mathcal{N}(0, c \mathbf{I})$ with some unknown variance $c$. The GLRTbased detector for this problem can then be written as (Kay, 1998, Theorem 9.1)

$$
T\left(\tilde{\mathbf{r}}_{z}\right) \triangleq \frac{\left(N^{\prime}-2\right)\left(\mathbf{D} \hat{\boldsymbol{\theta}}_{1}\right)^{T}\left[\mathbf{D}\left(\tilde{\mathbf{B}}^{T} \tilde{\mathbf{B}}\right)^{-1} \mathbf{D}^{T}\right]^{-1}\left(\mathbf{D} \hat{\boldsymbol{\theta}}_{1}\right)}{\tilde{\mathbf{r}}_{z}^{T}\left(\mathbf{I}-\tilde{\mathbf{B}}\left(\tilde{\mathbf{B}}^{T} \tilde{\mathbf{B}}\right)^{-1} \tilde{\mathbf{B}}^{T}\right) \tilde{\mathbf{r}}_{z}} \underset{\mathcal{H}_{0}}{\stackrel{\mathcal{H}_{1}}{\gtrless}} \gamma^{\prime}
$$

where $N^{\prime} \triangleq \frac{M(M+1)}{2}$ denotes the number of distinct equations, $\mathbf{D} \triangleq\left[\begin{array}{ll}0 & 1\end{array}\right]$, and $\hat{\boldsymbol{\theta}}_{1}=$ $\left(\tilde{\mathbf{B}}^{T} \tilde{\mathbf{B}}\right)^{-1} \tilde{\mathbf{B}}^{T} \tilde{\mathbf{r}}_{z}$ is the maximum likelihood estimate of $\boldsymbol{\theta}$ under $\mathcal{H}_{1}$. Furthermore, the probability of false alarm $\left(P_{\mathrm{FA}}\right)$ and probability of detection $\left(P_{\mathrm{D}}\right)$ of the approximate GLRT-based detector are given by

$$
\begin{gathered}
P_{\mathrm{FA}}=Q_{F_{1, N^{\prime}-2}}\left(\gamma^{\prime}\right), \\
P_{\mathrm{D}}=Q_{F_{1, N^{\prime}-2}(\lambda)}\left(\gamma^{\prime}\right),
\end{gathered}
$$

where the noncentrality parameter takes the form

$$
\lambda=\frac{\left(\mathbf{D} \boldsymbol{\theta}_{1}\right)^{T}\left[\mathbf{D}\left(\tilde{\mathbf{B}}^{T} \tilde{\mathbf{B}}\right)^{-1} \mathbf{D}^{T}\right]^{-1}\left(\mathbf{D} \boldsymbol{\theta}_{1}\right)}{c},
$$

where $\boldsymbol{\theta}_{1}$ is the true value of $\boldsymbol{\theta}$ under $\mathcal{H}_{1}$. 


\section{Extension to Frequency-Selective Fading Channels}

In this section we extend the results obtained in the previous sections to the case of wideband frequency-selective channel. Assume that the multipath channel between the PU transmitter and the SU receiver has the model $H(z)=h_{0}+h_{1} z^{-1}+\ldots+h_{L} z^{-L}$. Putting the tap weights in $(L+1) \times 1$ vector $\mathbf{h} \triangleq\left[h_{0}, h_{1}, \ldots, h_{L}\right]^{T}$ and denoting the $k$-th OFDM block of length $T_{\text {sym }}=N$ as $\mathbf{s}_{k} \triangleq\left[s_{k}(1), s_{k}(2), \ldots, s_{k}(N)\right]$ where $s_{k}(n) \triangleq s[(k-1) N+n]$, we have

$$
\mathbf{x}_{k}=\mathbf{S}_{k} \mathbf{h}+\mathbf{n}, k=1,2, \ldots, N_{B}
$$

where $\mathbf{S}_{k}$ is a Toeplitz $N \times(L+1)$ matrix defined as

$$
\mathbf{S}_{k} \triangleq\left[\begin{array}{ccccc}
s_{k}(1) & s_{k-1}(N) & s_{k-1}(N-1) & \ldots & s_{k-1}(N-L+1) \\
s_{k}(2) & s_{k}(1) & s_{k-1}(N) & \ldots & s_{k-1}(N-L+2) \\
s_{k}(3) & s_{k}(2) & s_{k}(1) & \ldots & s_{k-1}(N-L+3) \\
\ldots & \ldots & \ldots & \ldots & \ldots \\
s_{k}(N) & s_{k}(N-1) & s_{k}(N-2) & \ldots & s_{k}(N-L)
\end{array}\right]
$$

Denoting the $i$-th column of $\mathbf{S}_{k}$ by $\mathbf{s}_{k, i}$ we can write

$$
\mathbf{s}_{k, i}=\mathbf{J}_{d}^{i} \mathbf{s}_{k}+\mathbf{J}_{u}^{N-i} \mathbf{s}_{k-1}
$$

where $\mathbf{J}_{d}$ and $\mathbf{J}_{u}$ are respectively down-shift matrix and up-shift matrix whose $(i, j)$-th

elements are $\left[\mathbf{J}_{d}\right]_{i, j} \triangleq \delta_{i, j+1}$ and $\left[\mathbf{J}_{u}\right]_{i, j} \triangleq \delta_{i+1, j}$ where $\delta_{i, j}$ denotes the Kronecker delta. Remark that $\mathbf{J}_{d}=\mathbf{J}_{u}^{T}$. 
From (Seber, 2008, Theorem 21.6) and since $\mathbb{E}\left(\mathbf{S}_{k}\right)=\mathbf{0}_{N, L+1}$ we can next write

$$
\begin{aligned}
\mathbf{R}_{x} & =\mathbb{E}\left(\mathbf{S}_{k} \mathbf{h} \mathbf{h}^{H} \mathbf{S}_{k}^{H}\right)+\sigma_{0}^{2} \mathbf{I} \\
& =\sum_{i=0}^{L} \sum_{j=0}^{L} h_{i} h_{j}^{*} \operatorname{Cov}\left(\mathbf{s}_{k, i}, \mathbf{s}_{k, j}\right)+\sigma_{0}^{2} \mathbf{I} .
\end{aligned}
$$

From (31), $\operatorname{Cov}\left(\mathbf{s}_{k, i}, \mathbf{s}_{k, j}\right)$ can be computed as

$$
\operatorname{Cov}\left(\mathbf{s}_{k, i}, \mathbf{s}_{k, j}\right)=\sigma_{s}^{2}\left(\mathbf{J}_{d}^{i}(\boldsymbol{\Lambda}+\mathbf{I}) \mathbf{J}_{u}^{j}+\mathbf{J}_{u}^{N-i}(\boldsymbol{\Lambda}+\mathbf{I}) \mathbf{J}_{d}^{N-j}\right)
$$

Denoting $\mathbf{c}_{i, j} \triangleq \operatorname{vec}\left(\mathbf{J}_{d}^{i}(\boldsymbol{\Lambda}+\mathbf{I}) \mathbf{J}_{u}^{j}+\mathbf{J}_{u}^{N-i}(\boldsymbol{\Lambda}+\mathbf{I}) \mathbf{J}_{d}^{N-j}\right)$, we can write

$$
\begin{aligned}
\operatorname{vec}\left(\mathbf{R}_{x}\right) & =\sum_{i=0}^{L} \sum_{j=0}^{L} h_{i} h_{j}^{*} \mathbf{c}_{i, j}+\sigma_{0}^{2} \operatorname{vec}(\mathbf{I}) \\
& =\left[\mathbf{C}_{s}, \operatorname{vec}(\mathbf{I})\right]\left[\begin{array}{c}
\tilde{\mathbf{h}} \\
\sigma_{0}^{2}
\end{array}\right],
\end{aligned}
$$

where $\mathbf{C}_{s}$ is defined as the $N^{2} \times(L+1)^{2}$ matrix whose $(j(L+1)+i+1)$-th column is $\mathbf{c}_{i, j}$ (remark that $0 \leq i, j \leq L)$ and $\tilde{\mathbf{h}}$ is defined as the vector whose $(j(L+1)+i+1)$-th entry is $\sigma_{s}^{2} h_{i} h_{j}^{*}$.

Putting (34) in (18) and vectorizing the resulting equation yields

$$
\overline{\mathbf{r}}_{z}=\mathbf{B}_{m} \boldsymbol{\theta}_{m}+\mathbf{v}
$$

where $\mathbf{B}_{m} \triangleq \boldsymbol{\Omega}_{M}(\mathbf{A} \otimes \mathbf{A})\left[\mathbf{C}_{s}, \operatorname{vec}(\mathbf{I})\right], \boldsymbol{\theta}_{m} \triangleq\left[\tilde{\mathbf{h}}^{T}, \sigma_{0}^{2}\right]^{T}$, and $\mathbf{v} \triangleq \boldsymbol{\Omega}_{M}(\mathbf{A} \otimes \mathbf{A}) \boldsymbol{\Gamma}_{N} \operatorname{vech}(\mathbf{W})$. Then the problem of OFDM detection can be expressed as performing the following test for 
the linear model of (35)

$$
\left\{\begin{array}{l}
\mathcal{H}_{0}: \mathbf{D}_{m} \boldsymbol{\theta}_{m}=\mathbf{0} \\
\mathcal{H}_{1}: \mathbf{D}_{m} \boldsymbol{\theta}_{m} \neq \mathbf{0}
\end{array}\right.
$$

where $\mathbf{D}_{m}=\left[\mathbf{I}_{(L+1)^{2}}, \mathbf{0}_{(L+1)^{2}, 1}\right]$. The rest of the test formulation is similar to the Gaussian case discussed in 4 .

\section{Simulation Examples}

In this section we study the performance of the proposed sub-Nyquist OFDM detector by simulation examples. For simplicity, we consider an OFDM system with IFFT size 32 which means $T=32$. The cyclic prefix length is chosen as $T_{c p}=T / 4=8$ and subcarrier symbols are assumed to be drawn from a 16-QAM constellation with unit energy. The elements of the measurement matrix $\mathbf{A}$ are drawn from $\mathcal{N}(0,1)$ and its columns are normalized to have unit norm. We emphasize that these chosen values are just examples for carrying out numerical simulations, and are not as such related to the fundamentals of the derived detectors in any way.

Before proceeding with the simulation examples, we briefly discuss the parameters which affect the performance based on the formulation of the problem given in previous sections. We first recall that the linear equation used for detecting the OFDM signal is (18). Therefore the parameters which affect the detection are those affecting the solution of this 2-D linear regression problem. The first parameter is the number of independent equations in (18), i.e., $M(M+1) / 2$. For a fixed Nyquist frame size $N$, this parameter is uniquely specified by the compression ratio $\rho$. The second and third parameters are those which affect the power of finite sample error W. Formulas (16)-(17) clearly show that, for a given signal power $\sigma_{s}^{2}$, this

is determined by $N_{B}$; the number of blocks taken for computing $\mathbf{R}_{z}$, and $\sigma_{n}^{2}$; the variance 
of noise, or equivalently, signal to noise ratio. The first two experiments in this section are devised based on the above observations. Besides, the exactness of the approximation we adopted in Section 4 will be studied by simulation results. As mentioned in Section 1 there is no method in the literature specifically designed for single-band OFDM detection from sub-Nyquist samples. Therefore, to compare our method with some existing methods, we choose the method proposed in (Tian et al., 2012, Section IV) which recovers the PSD of a general stationary signal from sub-Nyquist samples.

The third experiment studies the performance of the method over frequency-selective channels.

\subsection{Influence of the compression ratio}

In the first experiment we study the effect of compression ratio on detection performance. For this experiment, the detector deploys $N_{B}=100$ OFDM symbols. Figure 11illustrates the probability of detection of the proposed method as a function of signal-to-noise ratio (SNR) for different compression ratios $\rho \in\{0.2,0.4,0.6,0.8,1\}$ when the threshold is set to obtain $P_{\mathrm{FA}}=0.05$. To check also the exactness of the approximation adopted in Section 4, we compute the threshold $\gamma^{\prime}$ both from the true $P_{F A}$ calculated from the simulated data in the absence of OFDM signal (solid lines with circle markers) and from formula (26) (dotted lines

with diamond marker). The results of the PSD recovery method introduced in Tian et al. (2012) are shown by dashed lines with square markers. For this method the threshold has been computed from the true $P_{F A}$.

The first observation from 1 is that, as we expect, the performance of the proposed method enhances with increasing the compression ratio. Interestingly, with heavy compression ratio of 0.2 , the detection probability is still above $95 \%$ at an SNR of $0 \mathrm{~dB}$. Notice that since the number of independent covariance-based equations is of order $\mathcal{O}\left(M^{2}\right)$, a compression ratio 
of $\rho \triangleq M / N=0.2$ implies that the effective compression ratio (in covariance domain) is only about $0.2^{2}=0.04$ or $4 \%$. Also, as it can be observed, the proposed method provides a much better performance compared to the general PSD recovery method of (Tian et al., 2012, Section IV). This can be seen also from Figure2 where the Receiver Operating Characteristic (ROC) curves of both methods have been depicted.

Furthermore, the unnoticeable difference between the curves with threshold computed from true $P_{F A}$ and the ones with threshold computed from (26) in Figure 1 verifies the exactness of the approximation adopted in Section 4 .

\subsection{Influence of the number of blocks}

In the second experiment we study the effect of number of blocks taken for computing the covariance matrix, $N_{B}$, on detection performance. As it can be seen from Figure 3 increasing $N_{B}$ improves the performance of the detector. In fact, in asymptotic case when $N_{B} \rightarrow \infty$

we have $\overline{\mathbf{R}}_{x}=\frac{1}{N_{B}} \sum_{l=1}^{N_{B}}\left(\mathbf{x}(l) \mathbf{x}(l)^{H}\right)=\mathbb{E}\left(\mathbf{x x}^{H}\right)=\mathbf{R}_{x}$ which means $\mathbf{W}=\mathbf{0}$. Similar to the previous experiment, it can be also observed that the performance of the proposed method shows significance improvement over the PSD recovery method of Tian et al. (2012). This can be seen also from Fiure 4 where the ROC curves of both methods have been illustrated.

Again, the unnoticeable difference between the solid lines (true $P_{F A}$ ) and their corresponding dotted lines (target $P_{F A}$ ) in Figure 3 confirms the exactness of the approximation we used in Section 4 . 


\subsection{Performance of the proposed method over frequency-selective channels}

The third experiment studies the effect of frequency-selective channels on the performance of the proposed method. The method is applied on three different channel lengths with identical

energy; i.e. $\sum_{l=0}^{L}\left|h_{l}\right|^{2}$ is identical for all $L=0,1,2$. The OFDM signal characteristics as well as the probability of false-alarm are set to the same values as in the two previous examples. As it can be observed from Figure 5 the frequency selectivity slightly deteriorates the performance but reliable sensing can still be achieved even at low SNRs.

\section{Concluding Remarks}

A method for sensing OFDM signals from sub-Nyquist samples was proposed. The proposed method exploits the unique characteristics of the covariance matrix of OFDM signal to perform the spectrum sensing task. Based on the statistical properties of the covariance matrix we developed an approximate GLRT-based detector. The proposed method was also extended to the case of frequency-selective channels. The simulation results verify the theoretical observations. The results also illustrate that highly efficient sensing can still be obtained, in terms of probability of detection and false alarm, despite of low compression ratios and low SNRs. This can open up new possibilities for sensing devices with low-cost analog hardware and A/D interface deploying sub-Nyquist observations.

\section{Acknowledgments}

The work of S. A. Razavi and M. Valkama was supported by the Academy of Finland under the project \#251138 "Digitally-Enhanced RF for Cognitive Radio Devices" and the 
Finnish Funding Agency for Technology and Innovation (Tekes), under the project "Enabling Methods for Dynamic Spectrum Access and Cognitive Radio (ENCOR)".

\section{Appendix: Proof of Theorem 1}

The proof of (16) is trivial. Here we only prove (17). Let us define $r_{i, j} \triangleq \operatorname{Real}\left(\left[\overline{\mathbf{R}}_{x}\right]_{i, j}\right)$, and denote the real part of $x_{i}$ by $\dot{x}_{i}$ and its imaginary part by $\grave{x}_{i}$. Since

$$
\begin{aligned}
r_{i, j} & =\operatorname{Real}\left(\frac{1}{N_{B}} \sum_{l=1}^{N_{B}}\left(x_{i}(l) x_{j}^{*}(l)\right)\right) \\
& =\frac{1}{N_{B}} \sum_{l=1}^{N_{B}}\left(\grave{x}_{i}(l) \dot{x}_{j}(l)+\grave{x}_{i}(l) \grave{x}_{j}(l)\right),
\end{aligned}
$$

we can write

$$
\begin{aligned}
r_{i, j} r_{p, q}= & \frac{1}{N_{B}{ }^{2}} \sum_{l=1}^{N_{B}} \sum_{l^{\prime}=1}^{N_{B}}\left(\left[\dot{x}_{i}(l) \dot{x}_{j}(l)+\grave{x}_{i}(l) \grave{x}_{j}(l)\right]\right. \\
& {\left.\left[\grave{x}_{p}\left(l^{\prime}\right) \dot{x}_{q}\left(l^{\prime}\right)+\grave{x}_{p}\left(l^{\prime}\right) \grave{x}_{q}\left(l^{\prime}\right)\right]\right) } \\
= & T_{1}+T_{2},
\end{aligned}
$$

where $T_{1}$ and $T_{2}$ are defined as follows

$$
\begin{aligned}
T_{1} \triangleq & \frac{1}{N_{B}{ }^{2}} \sum_{l=1}^{N_{B}} \sum_{\substack{l^{\prime}=1 \\
l^{\prime} \neq l}}^{N_{B}}\left(\left[\dot{x}_{i}(l) \dot{x}_{j}(l)+\grave{x}_{i}(l) \grave{x}_{j}(l)\right]\right. \\
& {\left.\left[\dot{x}_{p}\left(l^{\prime}\right) \dot{x}_{q}\left(l^{\prime}\right)+\grave{x}_{p}\left(l^{\prime}\right) \grave{x}_{q}\left(l^{\prime}\right)\right]\right) } \\
T_{2} \triangleq & \frac{1}{N_{B}{ }^{2}} \sum_{l=1}^{N_{B}}\left(\left[\dot{x}_{i}(l) \dot{x}_{j}(l)+\grave{x}_{i}(l) \grave{x}_{j}(l)\right]\right. \\
& {\left.\left[\dot{x}_{p}(l) \dot{x}_{q}(l)+\grave{x}_{p}(l) \grave{x}_{q}(l)\right]\right) }
\end{aligned}
$$

The reason for partitioning (37) to $T_{1}$ and $T_{2}$ is that in $T_{1}$ the two multiplicative terms inside the double summation are independent since they belong to different frames $l$ and $l^{\prime}$, 
while in $T_{2}$ they belong to the same frame and depending on indices $i, j, p$, and $q$ might be dependent. Due to independence of multiplicative terms, $\mathbb{E}\left(T_{1} \mid \mathcal{H}_{1}\right)$ is easy to compute as

$$
\mathbb{E}\left(T_{1} \mid \mathcal{H}_{1}\right)= \begin{cases}\frac{N_{B}-1}{N_{B}} \tau_{0}^{2} & \text { if }(i-j, p-q)=(0,0) \\ \frac{N_{B}-1}{N_{B}} \tau_{0} \tau_{s} & \text { if }(i-j, p-q) \in\{(0, T),(T, 0)\} \\ \frac{N_{B}-1}{N_{B}} \tau_{s}^{2} & \text { if }(i-j, p-q)=(T, T) \\ 0 & \text { otherwise. }\end{cases}
$$

Furthermore, after some more scrutinized manipulations, $\mathbb{E}\left(T_{2} \mid \mathcal{H}_{1}\right)$ can be expressed as

$$
\mathbb{E}\left(T_{2} \mid \mathcal{H}_{1}\right)= \begin{cases}\frac{2}{N_{B}} \tau_{0}^{2} & \text { if }(i, j)=(p, q), i-j=0, \\ \frac{1}{2 N_{B}}\left(\tau_{0}^{2}+3 \tau_{s}^{2}\right) & \text { if }(i, j)=(p, q), i-j=T, \\ \frac{1}{2 N_{B}} \tau_{0}^{2} & \text { if }(i, j)=(p, q), i-j \notin\{0, T\}, \\ \frac{2}{N_{B}} \tau_{0} \tau_{s} & \text { if } i=j=p=q+T, \\ \frac{1}{N_{B}} \tau_{0}^{2} & \text { if }(i-j, p-q)=(0,0), i \neq p \\ \frac{1}{N_{B}} \tau_{s}^{2} & \text { if }(i-j, p-q)=(T, T), i \neq p \\ \frac{1}{N_{B}} \tau_{0} \tau_{s} & \text { if }(i-j, p-q) \in\{(0, T),(T, 0)\}, \\ & \text { otherwise. }\end{cases}
$$


Summing (38) and (39) will yield then

$$
\mathbb{E}\left(r_{i, j} r_{p, q} \mid \mathcal{H}_{1}\right)= \begin{cases}\tau_{0}^{2}+\frac{\tau_{0}^{2}}{N_{B}} & \text { if }(i, j)=(p, q), i-j=0, \\ \tau_{s}^{2}+\frac{\tau_{0}^{2}+\tau_{s}^{2}}{2 N_{B}} & \text { if }(i, j)=(p, q), i-j=T, \\ \frac{\tau_{0}^{2}}{2 N_{B}} & \text { if }(i, j)=(p, q), i-j \notin\{0, T\}, \\ \tau_{0} \tau_{s}+\frac{\tau_{0} \tau_{s}}{N_{B}} & \text { if } i=j=p=q+T, \\ \tau_{0}^{2} & \text { if }(i-j, p-q)=(0,0), i \neq p \\ \tau_{s}^{2} & \text { if }(i-j, p-q)=(T, T), i \neq p \\ \tau_{0} \tau_{s} & \text { if }(i-j, p-q) \in\{(0, T), \\ & \text { otherwise. }\end{cases}
$$

Subtracting $\mathbb{E}\left(r_{i, j} \mid \mathcal{H}_{1}\right) \mathbb{E}\left(r_{p, q} \mid \mathcal{H}_{1}\right)$ from (40) and remarking that $w_{i, j}$ and $r_{i, j}$ have identical variances, (17) is concluded.

\section{References}

M. Adrat, J. Leduc, S. Couturier, M. Antweiler, and H. Elders-Boll. 2nd order cyclostationarity of ofdm signals: Impact of pilot tones and cyclic prefix. In IEEE ICC 2009, pages 1-5, June 2009.

Ala' Al-Habashna, Octavia A Dobre, Ramachandran Venkatesan, and Dimitrie C Popescu. Second-order cyclostationarity of mobile wimax and lte ofdm signals and application to spectrum awareness in cognitive radio systems. Selected Topics in Signal Processing, IEEE Journal of, 6(1):26-42, 2012.

Dyonisius Dony Ariananda and Geert Leus. Wideband power spectrum sensing using sub- 
nyquist sampling. In Signal Processing Advances in Wireless Communications (SPAWC), 2011 IEEE 12th International Workshop on, pages 101-105. IEEE, 2011.

E. Axell and E. G. Larsson. Optimal and sub-optimal spectrum sensing of ofdm signals in known and unknown noise variance. Selected Areas in Communications, IEEE Journal on, 29(2):290-304, 2011.

Simin Bokharaiee, Ha H Nguyen, and Ed Shwedyk. Blind spectrum sensing for ofdm-based cognitive radio systems. Vehicular Technology, IEEE Transactions on, 60(3):858-871, 2011.

S. Chaudhari, V. Koivunen, and H. V. Poor. Autocorrelation-based decentralized sequential detection of ofdm signals in cognitive radios. Signal Processing, IEEE Transactions on, 57(7):2690-2700, 2009.

D. Cohen, E. Rebeiz, V. Jain, Y. C. Eldar, and D. Cabric. Cyclostationary feature detection from sub-Nyquist samples. In IEEE CAMSAP, pages 333-336, 2011.

A. S. Goldberger. A Course in Econometrics. Harvard University Press, 1991.

S. Haykin, D.J. Thomson, and J.H. Reed. Spectrum sensing for cognitive radio. Proceedings of the IEEE, 97(5):849-877, May 2009.

S.M. Kay. Fundamentals of statistical signal processing: detection theory. Prentice Hall, 1998.

Sami Kirolos, Jason Laska, Michael Wakin, Marco Duarte, Dror Baron, Tamer Ragheb, Yehia Massoud, and Richard Baraniuk. Analog-to-information conversion via random demodulation. In Design, Applications, Integration and Software, 2006 IEEE Dallas/CAS Workshop on, pages 71-74. IEEE, 2006.

Geert Leus and Zhi Tian. Recovering second-order statistics from compressive measurements. 
In Computational Advances in Multi-Sensor Adaptive Processing (CAMSAP), 2011 4th IEEE International Workshop on, pages 337-340. IEEE, 2011.

M. Mishali and Y.C. Eldar. From theory to practice: Sub-Nyquist sampling of sparse wideband analog signals. Selected Topics in Signal Processing, IEEE Journal of, 4(2):375 -391, April 2010.

M. Mishali and Y.C. Eldar. Wideband spectrum sensing at sub-Nyquist rates. Signal Processing Magazine, IEEE, 28(4):102 -135, July 2011.

Moshe Mishali, Yonina C Eldar, and Asaf J Elron. Xampling: Signal acquisition and processing in union of subspaces. Signal Processing, IEEE Transactions on, 59(10):4719-4734, 2011.

J. Mitola and G. Q. Maguire. Cognitive radio: making software radios more personal. Personal Communications, IEEE, 6(4):13 -18, Aug. 1999.

Y.L. Polo, Y. Wang, A. Pandharipande, and G. Leus. Compressive wide-band spectrum sensing. In IEEE International Conference on Acoustics, Speech and Signal Processing, 2009., pages 2337 -2340, Apr. 2009.

S. A. Razavi, M. Valkama, and D. Cabric. High-resolution cyclic spectrum reconstruction from sub-Nyquist samples. In IEEE Workshop on Signal rocessing Advances in Wireless Communications (IEEE SPAWC 2014), pages 250-254, June 16-19 2013.

S. A. Razavi, M. Valkama, and D. Cabric. Signature-assisted rendezvous in ofdm-based cognitive networks using sub-Nyquist samples. In Proc. 8th IEEE International Workshop on Sensor Array and Multichannel Signal Processing, (IEEE SAM 2014), pages 401-404, June 22-25 2014.

E. Rebeiz, V. Jain, and D. Cabric. Cyclostationary-based low complexity wideband spectrum 
sensing using compressive sampling. In International Conference on Communications (ICC 2012), 2012.

G. A. F. Seber. A Matrix Handbook for Statisticians. Wiley-Interscience, 2008.

H. Sun, W. Chiu, J. Jiang, A. Nallanathan, and H. V. Poor. Wideband spectrum sensing with sub-Nyquist sampling in cognitive radios. Signal Processing, IEEE Transactions on, 60(11):6068-6073, Nov. 2012.

Hongjian Sun, Wei-Yu Chiu, Jing Jiang, Arumugam Nallanathan, and H Vincent Poor. Wideband spectrum sensing with sub-nyquist sampling in cognitive radios. arXiv preprint arXiv:1302.1847, 2013.

P. D. Sutton, K. E. Nolan, and L. E. Doyle. Cyclostationary signatures in practical cognitive radio applications. Selected Areas in Communications, IEEE Journal on, 26(1):13-24, Jan. 2008.

Z. Tian and G.B. Giannakis. Compressed sensing for wideband cognitive radios. In IEEE International Conference on Acoustics, Speech and Signal Processing, 200\%., pages IV1357 -IV-1360, April 2007.

Z. Tian, Y. Tafesse, and B. Sadler. Cyclic feature detection with sub-Nyquist sampling for wideband spectrum sensing. Selected Topics in Signal Proc., IEEE Journal of, 6(1):58 -69, Feb. 2012.

Zhi Tian. Compressed wideband sensing in cooperative cognitive radio networks. In Global Telecommunications Conference, 2008. IEEE GLOBECOM 2008. IEEE, pages 1-5. IEEE, 2008.

Zhi Tian. Cyclic feature based wideband spectrum sensing using compressive sampling. In Communications (ICC), 2011 IEEE International Conference on, pages 1-5. IEEE, 2011. 
Joel A Tropp, Jason N Laska, Marco F Duarte, Justin K Romberg, and Richard G Baraniuk. Beyond nyquist: Efficient sampling of sparse bandlimited signals. Information Theory, IEEE Transactions on, 56(1):520-544, 2010.

Tevfik Yucek and Hüseyin Arslan. A survey of spectrum sensing algorithms for cognitive radio applications. Communications Surveys \& Tutorials, IEEE, 11(1):116-130, 2009. 


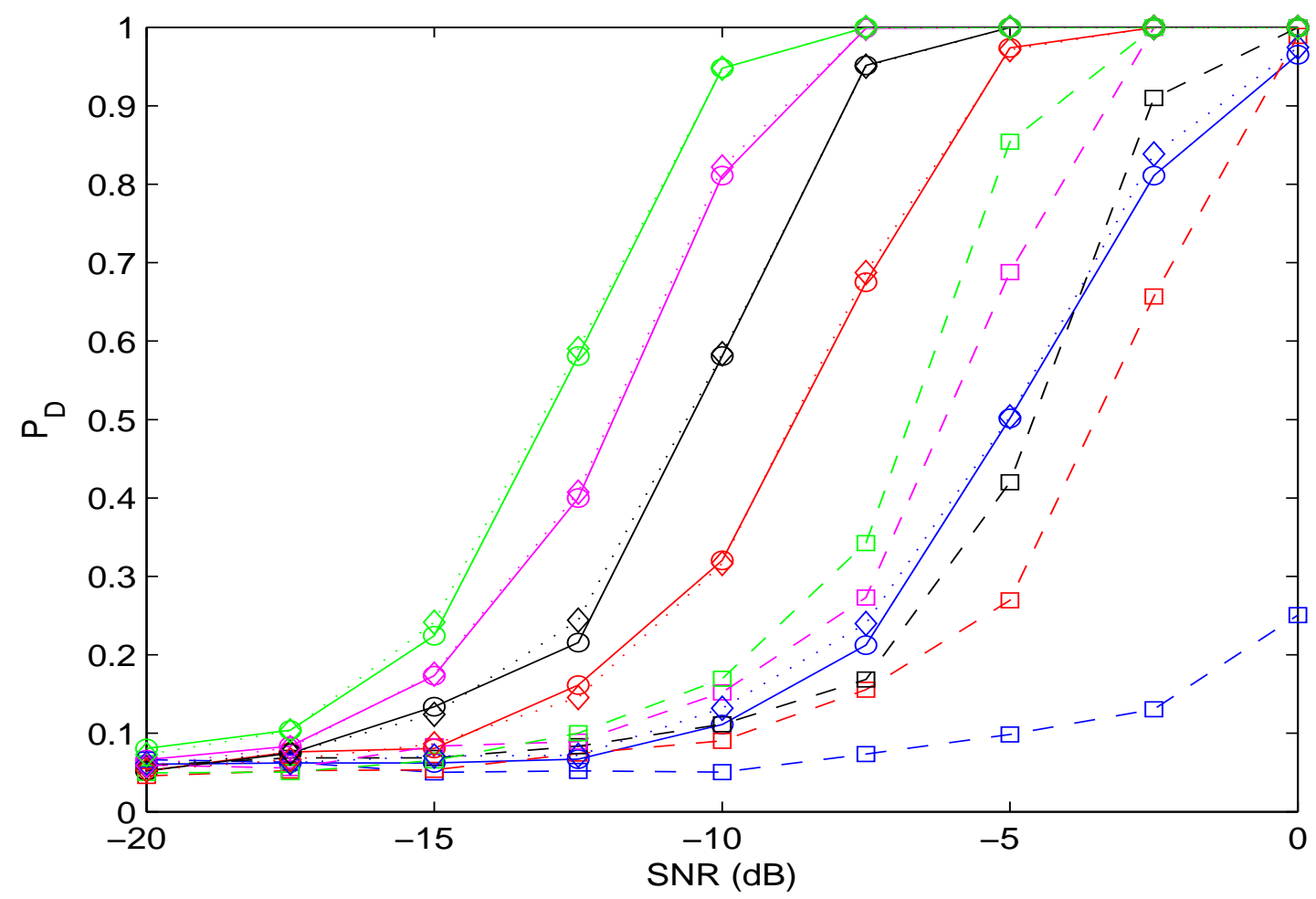

Figure 1: Probability of Detection versus SNR for various compression ratios $\rho$. The number of blocks is $N_{B}=100$ and the probability of false-alarm is $P_{\mathrm{FA}}=0.05$. The solid curves with circle markers correspond to the case where the threshold has been determined based on the true $P_{F A}$ computed from the simulated data. The dotted curves with diamond markers correspond to the case where the threshold has been determined from formula (26). The dashed curves with square markers correspond to the PSD recovery method introduced in Tian et al. (2012). Color conventions are as follows. Blue: $\rho=0.2$, red: $\rho=0.4$, black: $\rho=0.6$, magenta: $\rho=0.8$, green: $\rho=1$. As it can be seen the proposed algorithm clearly outperforms the method introduced in Tian et al. (2012). Furthermore, the very small distance between the solid curves with circle markers and the dotted curves with diamond markers shows the exactness of the approximation we adopted. 


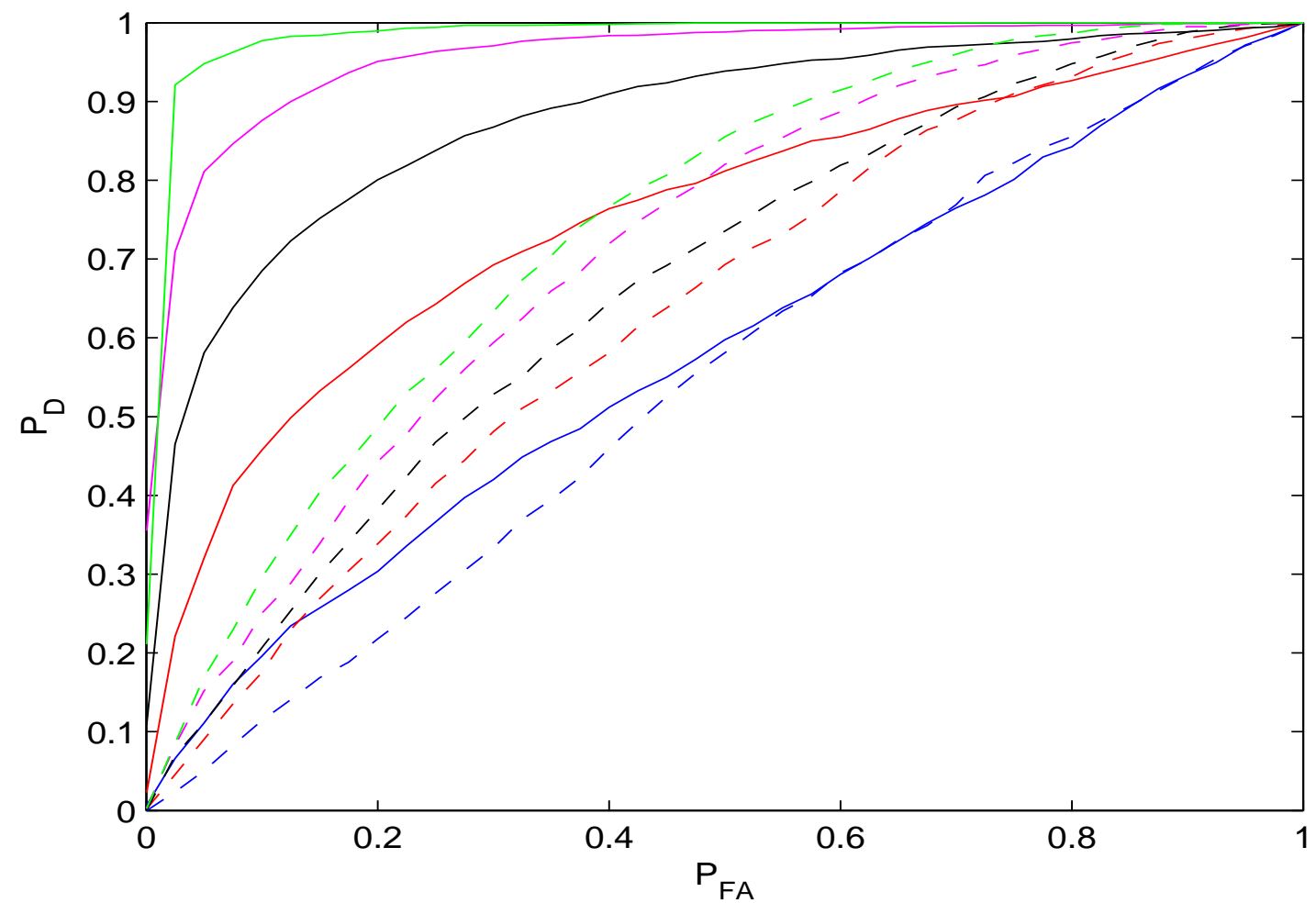

Figure 2: Probability of Detection versus SNR for various compression ratios $\rho$. The number of blocks is $N_{B}=100$ and the probability of false-alarm is $P_{\mathrm{FA}}=0.05$. The solid curves correspond to the case where the threshold has been determined based on the true $P_{F A}$ computed from the simulated data. The dashed curves correspond to the PSD recovery method introduced in Tian et al. (2012). The color conventions are as in Figure 1. As it can be seen the proposed algorithm clearly outperforms the method introduced in Tian et al. (2012). 


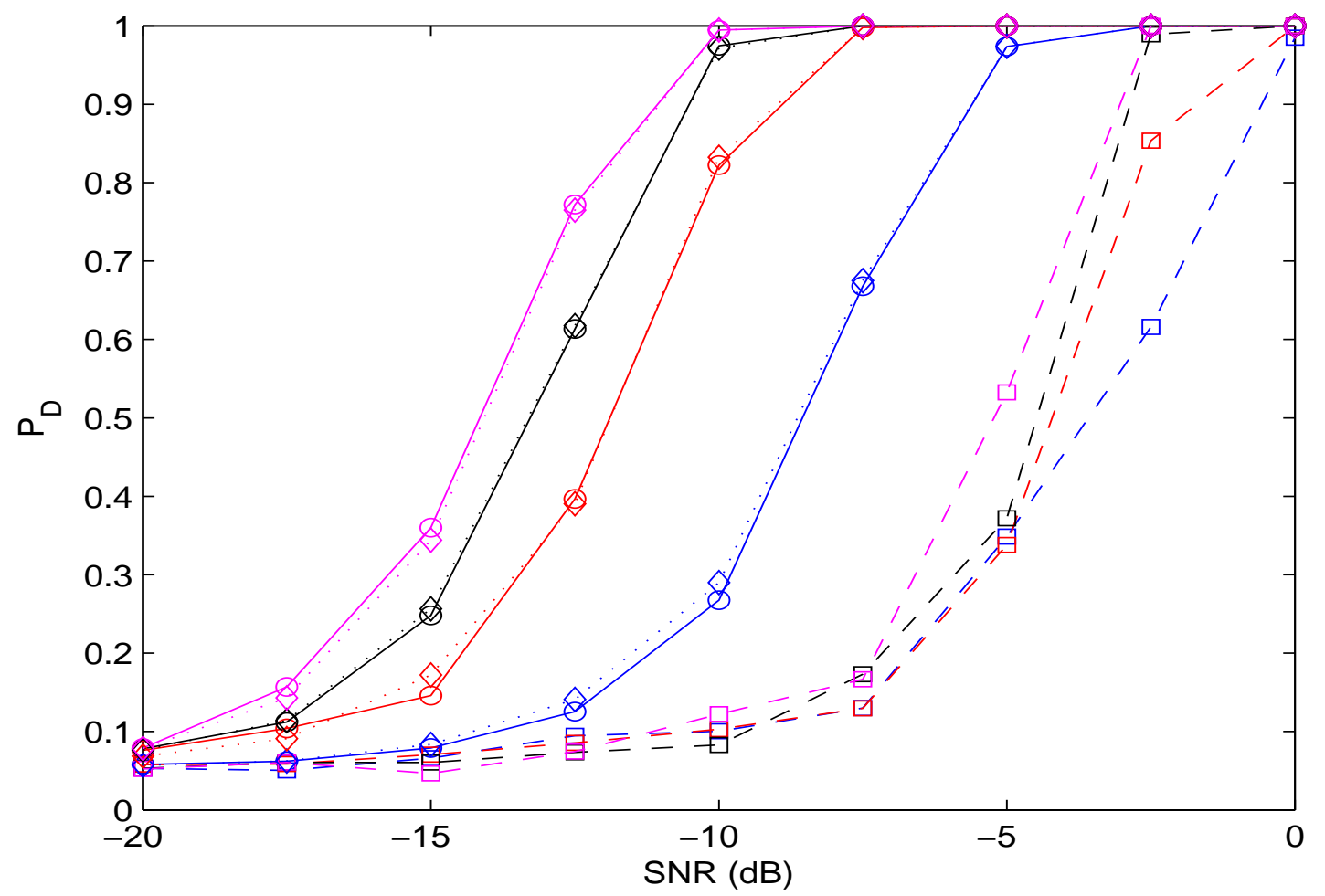

Figure 3: Probability of Detection versus SNR for various number of blocks $N_{B}$. The compression ratio is $\rho=0.4$ and the probability of false-alarm is $P_{\mathrm{FA}}=0.05$. The solid curves with circle markers correspond to the case where the threshold has been determined based on the true $P_{F A}$ computed from the simulated data. The dotted curves with diamond markers correspond to the case where the threshold has been determined from formula (26). The dashed curves with square markers correspond to the PSD recovery method introduced in Tian et al. (2012). Color conventions are as follows. Blue: $N_{B}=100$, red: $N_{B}=400$, black: $N_{B}=700$, magenta: $N_{B}=1000$. As it can be seen the proposed algorithm clearly outperforms the method introduced in Tian et al. (2012). Furthermore, the very small distance between the solid curves with circle markers and the dotted curves with diamond markers shows the exactness of the approximation we adopted. 


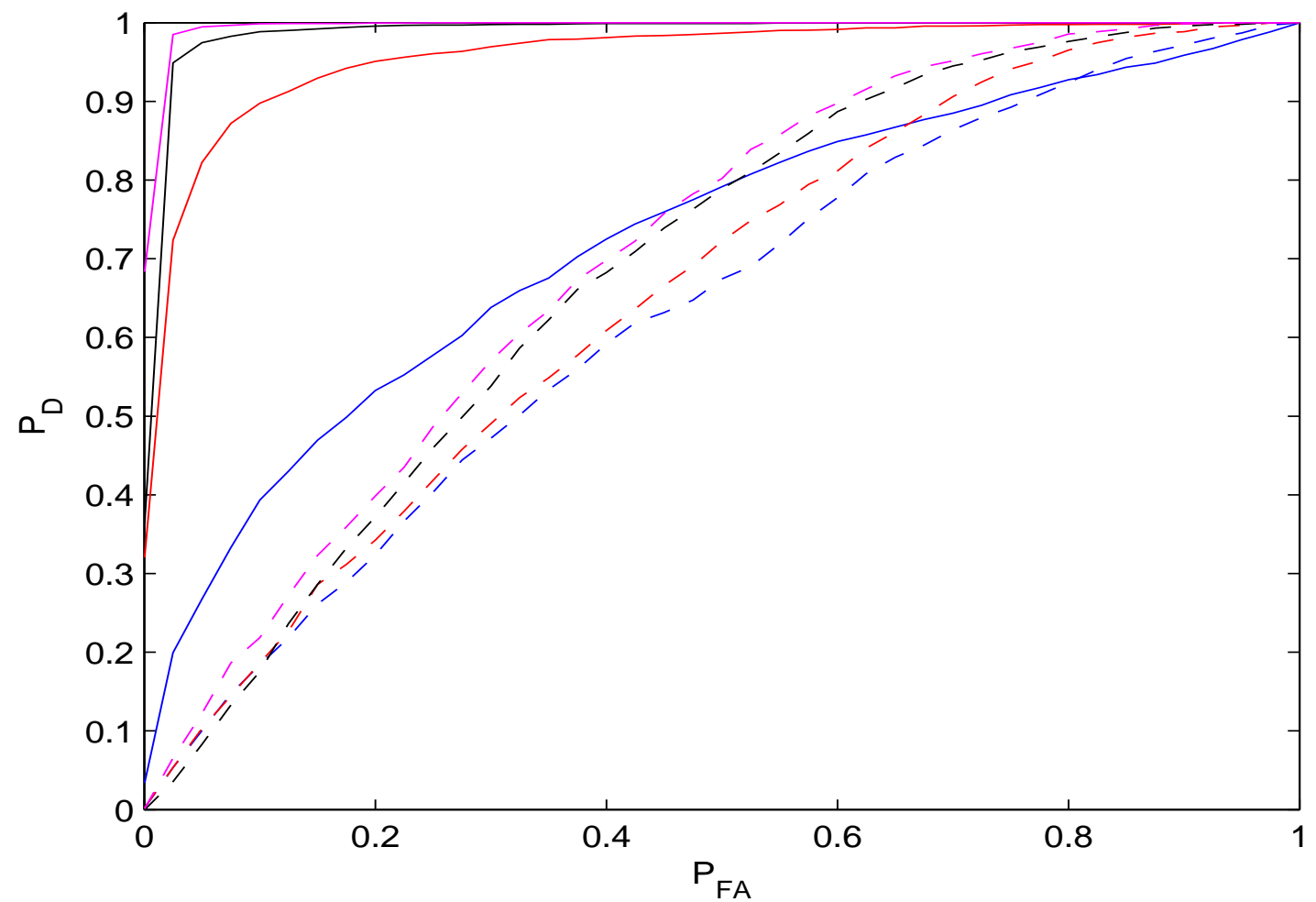

Figure 4: Probability of Detection versus SNR for various number of blocks $N_{B}$. The compression ratio is $\rho=0.4$ and the probability of false-alarm is $P_{\mathrm{FA}}=0.05$. The solid curves correspond to the case where the threshold has been determined based on the true $P_{F A}$ computed from the simulated data. The dashed curves correspond to the PSD recovery method introduced in Tian et al. (2012). The color conventions are as in Figure 3. As it can be seen the proposed algorithm clearly outperforms the method introduced in Tian et al. (2012). 


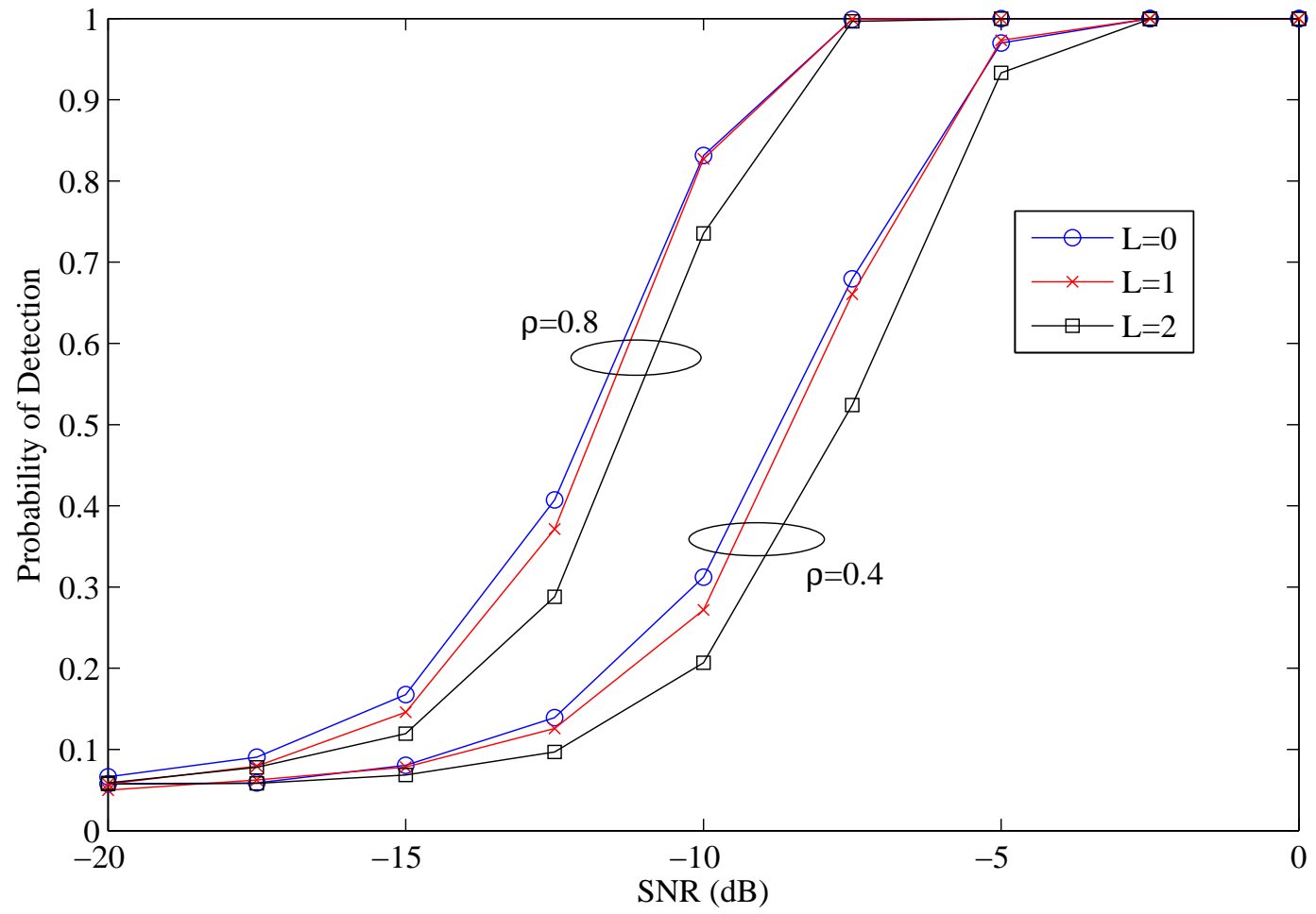

Figure 5: Effect of frequency-selectivity of the channels on the performance of the proposed method. The channel length is $L+1$ and $\rho$ is the compression ratio. 